\title{
ERRATUM: SIMPSON TYPE QUANTUM INTEGRAL INEQUALITIES FOR CONVEX FUNCTIONS
}

\author{
NECMETTIN ALP
}

Received 22 May, 2020

\begin{abstract}
We have shown that the results of [4] were wrong. Additionally, correct results concerning the Simpson type quantum integral inequalities are proved.
\end{abstract}

2010 Mathematics Subject Classification: 26D15, 26D10, 26A51, 34A08

Keywords: $q$-integral inequalities, $q$-derivative, Convex functions, Simpson's inequalities.

\section{INTRODUCTION}

In 2018 Tunç et al. [4] obtained Simpson's type quantum integral inequalities. Unfortunately, there are many mistakes in the proofs. Many $q$-integrals are calculated incorrectly. Besides, the results of lemma and theorems are also wrong. In this paper, we show the errors in the [4].

\section{PReliminaries AND Definitions of $q$-CAlCulus}

Throughout this paper, let $a<b$ and $0<q<1$ be a constant. The following definitions and theorems for $q$-derivative and $q$ - integral of a function $f$ on $[a, b]$ are given in $[2,3]$.

Definition 1. For a continuous function $f:[a, b] \rightarrow \mathbb{R}$ then $q$-derivative of $f$ at $x \in[a, b]$ is characterized by the expression

$$
{ }_{a} D_{q} f(x)=\frac{f(x)-f(q x+(1-q) a)}{(1-q)(x-a)}, x \neq a .
$$

Since $f:[a, b] \rightarrow \mathbb{R}$ is a continuous function, thus we have ${ }_{a} D_{q} f(a)=\lim _{x \rightarrow a}{ }_{a} D_{q} f(x)$. The function $f$ is said to be $q$ - differentiable on $[a, b]$ if ${ }_{a} D_{q} f(t)$ exists for all $x \in[a, b]$. If $a=0$ in (2.1), then ${ }_{0} D_{q} f(x)=D_{q} f(x)$, where $D_{q} f(x)$ is familiar $q$-derivative of $f$ at $x \in[a, b]$ defined by the expression (see [1])

$$
D_{q} f(x)=\frac{f(x)-f(q x)}{(1-q) x}, \quad x \neq 0 .
$$


Definition 2. Let $f:[a, b] \rightarrow \mathbb{R}$ be a continuous function. Then the $q$-definite integral on $[a, b]$ is delineated as

$$
\int_{a}^{x} f(t)_{a} d_{q} t=(1-q)(x-a) \sum_{n=0}^{\infty} q^{n} f\left(q^{n} x+\left(1-q^{n}\right) a\right)
$$

for $x \in[a, b]$.

If $a=0$ in (2.3), then $\int_{0}^{x} f(t)_{0} d_{q} t=\int_{0}^{x} f(t) d_{q} t$, where $\int_{0}^{x} f(t) d_{q} t$ is familiar $q$ definite integral on $[0, x]$ defined by the expression (see [1])

$$
\int_{0}^{x} f(t)_{0} d_{q} t=\int_{0}^{x} f(t) d_{q} t=(1-q) x \sum_{n=0}^{\infty} q^{n} f\left(q^{n} x\right) .
$$

If $c \in(a, x)$, then the $q$ - definite integral on $[c, x]$ is expressed as

$$
\int_{c}^{x} f(t)_{a} d_{q} t=\int_{a}^{x} f(t)_{a} d_{q} t-\int_{a}^{c} f(t)_{a} d_{q} t .
$$

$[n]_{q}$ notation

$$
[n]_{q}=\frac{q^{n}-1}{q-1}
$$

Lemma 1. [3] For $\alpha \in \mathbb{R} \backslash\{-1\}$, the following formula holds:

$$
\int_{a}^{x}(t-a)_{a}^{\alpha} d_{q} t=\frac{(x-a)^{\alpha+1}}{[\alpha+1]_{q}} .
$$

\section{ERRATUM: SIMPSON TYPE QUANTUM INTEGRAL INEQUALITIES FOR CONVEX FUNCTIONS}

Here, we will show the errors we mentioned above. For example, in Lemma 4 the followin equality is not correct:

$$
\begin{aligned}
\int_{0}^{\frac{1}{2}}(1-t)\left|q t-\frac{1}{6}\right|_{0} d_{q} t & =\int_{0}^{\frac{1}{2}}\left|q t-\frac{1}{6}\right|_{0} d_{q} t-\int_{0}^{\frac{1}{2}} t\left|q t-\frac{1}{6}\right|_{0} d_{q} t \\
& =\int_{0}^{\frac{1}{6 q}}\left(q t-\frac{1}{6}\right)_{0} d_{q} t+\int_{\frac{1}{6 q}}^{\frac{1}{2}}\left(\frac{1}{6}-q t\right)_{0} d_{q} t
\end{aligned}
$$




$$
-\left(\int_{0}^{\frac{1}{6 q}} t\left(q t-\frac{1}{6}\right)_{0} d_{q} t+\int_{\frac{1}{6 q}}^{\frac{1}{2}} t\left(\frac{1}{6}-q t\right)_{0} d_{q} t\right) .
$$

Here, for $q \in(0,1), \frac{1}{6 q} \not \leq \frac{1}{2}$. For instance, $q=\frac{1}{6} \rightarrow 1 \not \leq \frac{1}{2}$. So, the proof of Lemma 4 is not correct. Lemma 5 also have the same errors. On the other hand, since Lemma 4 and Lemma 5 are used in proof of Theorem 1, there are errors in this theorem. Moreover, Theorem 2 and 3 have the same mistakes. For instance, because of (2.6), the following equalities are also not true:

$$
\begin{aligned}
& \int_{0}^{\frac{1}{2}}\left|q t-\frac{1}{6}\right|_{0}^{p} d_{q} t=\frac{\left(1+(3 q-1)^{p+1}\right)(1-q)}{6^{p+1} q\left(1-q^{p+1}\right)}, \\
& \int_{\frac{1}{2}}^{1}\left|q t-\frac{5}{6}\right|_{0}^{p} d_{q} t=\frac{\left[(5-3 q)^{p+1}+(6 q-5)^{p+1}\right](1-q)}{6^{p+1} q\left(1-q^{p+1}\right)} .
\end{aligned}
$$

The integral boundaries that cause all these errors are chosen independently of $q$.

Now, let show the following Theorem 1 in [4] is not correct. For this, we give an example.

Theorem 1. Suppose that $f:[a, b] \rightarrow \mathbb{R}$ is a q-differentiable function on $(a, b)$ and $0<q<1$. If $\left|{ }_{a} D_{q} f\right|$ is convex and integrable function on $[a, b]$, then we possess the inequality

$$
\begin{aligned}
& \frac{1}{6}\left|f(a)+4 f\left(\frac{a+b}{2}\right)+f(b)-\frac{1}{(b-a)} \int_{a}^{b} f(t)_{a} d_{q} t\right| \\
& \quad \leq \frac{(b-a)}{12}\left\{\left.\frac{2 q^{2}+2 q+1}{q^{3}+2 q^{2}+2 q+1}\right|_{a} D_{q} f(b)\left|+\frac{1}{3} \frac{6 q^{3}+4 q^{2}+4 q+1}{q^{3}+2 q^{2}+2 q+1}\right|_{a} D_{q} f(a) \mid\right\} .
\end{aligned}
$$

Example 1. Let choose $f(t)=1-t$ on $[0,1]$ and $f(t)$ satisfies the conditions of Theorem 1. On the other hand, ${ }_{a} D_{q} f|=|{ }_{a} D_{q}(1-t) \mid=1$ is convex and integrable on $[0,1]$. Then we have

$$
\begin{aligned}
& \frac{1}{6}\left|f(a)+4 f\left(\frac{a+b}{2}\right)+f(b)-\frac{1}{(b-a)} \int_{a}^{b} f(t)_{a} d_{q} t\right| \\
& \quad=\frac{1}{6}\left|1+2+0-\int_{0}^{1}(1-t)_{0} d_{q} t\right|
\end{aligned}
$$




$$
=\frac{1}{6}\left|3-\left(t-\frac{t^{2}}{1+q}\right)_{0}^{1}\right|=\frac{3+2 q}{6(1+q)} \text {. }
$$

Also,

$$
\begin{aligned}
\frac{(b-a)}{12} & \left\{\frac{2 q^{2}+2 q+1}{q^{3}+2 q^{2}+2 q+1}\left|{ }_{a} D_{q} f(b)\right|+\left.\frac{1}{3} \frac{6 q^{3}+4 q^{2}+4 q+1}{q^{3}+2 q^{2}+2 q+1}\right|_{a} D_{q} f(a) \mid\right\} \\
& =\frac{1}{12}\left\{\frac{2 q^{2}+2 q+1}{q^{3}+2 q^{2}+2 q+1}+\frac{1}{3} \frac{6 q^{3}+4 q^{2}+4 q+1}{q^{3}+2 q^{2}+2 q+1}\right\} \\
& =\frac{1}{36} \frac{6 q^{2}+6 q+3+6 q^{3}+4 q^{2}+4 q+1}{q^{3}+2 q^{2}+2 q+1} \\
& =\frac{1}{36} \frac{6 q^{3}+10 q^{2}+10 q+4}{q^{3}+2 q^{2}+2 q+1} \\
& =\frac{1}{18} \frac{3 q^{3}+5 q^{2}+5 q+2}{q^{3}+2 q^{2}+2 q+1} .
\end{aligned}
$$

As we seen, from (3.2) and (3.3) and for $q \in(0,1)$ we write

$$
\frac{3+2 q}{6(1+q)} \not \leq \frac{1}{18} \frac{3 q^{3}+5 q^{2}+5 q+2}{q^{3}+2 q^{2}+2 q+1} \text {. }
$$

For instance, choosing $q=\frac{1}{2}$ we have

$$
\frac{4}{9} \not \leq \frac{7}{54} \text {. }
$$

Therefore, Inequality (3.1) is not correct.

Similarly, other theorems can be shown to be false.

\section{REFERENCES}

[1] V. Kac and P. Cheung, Quantum calculus. Springer-Verlag New York, 2001. doi: 10.1007/9781-4613-0071-7.

[2] J. Tariboon and S. K. Ntouyas, "Quantum calculus on finite intervals and applications to impulsive difference equations," Advances in Difference Equations, vol. 2013, no. 1, p. 282, 2013, doi: 10.1186/1687-1847-2013-282.

[3] J. Tariboon and S. K. Ntouyas, "Quantum integral inequalities on finite intervals," Journal of Inequalities and Applications, vol. 2014, no. 1, p. 121, 2014, doi: 10.1186/1029-242X-2014-121.

[4] M. Tunç, E. Gov, and S. Balgecti, "Simpson type quantum integral inequalities for convex functions," Miskolc Math. Notes, vol. 19, no. 1, pp. 649-664, 2018, doi: 10.18514/MMN.2018.1661.

\section{Author's address}

\section{Necmettin Alp}

Duzce University, Department of Mathematics, Faculty of Science, Konuralp, Duzce, Turkey

E-mail address: placennegmail.com 